OPEN ACCESS

Edited by:

Thandavarayan Ramamurthy, National Institute of Cholera and

Enteric Diseases (ICMR), India

Reviewed by:

Durg Vijai Singh,

Institute of Life Sciences (ILS), India

M. Jahangir Alam,

University of Houston, United States

*Correspondence:

Rama Chaudhry

drramach@gmail.com

${ }^{\dagger}$ Present address:

Bhaskar Thakur:

Division of Biostatistics and Epidemiology, Texas Tech Health

Science Center, El Paso, TX, United States

Specialty section:

This article was submitted to

Environmental Health,

a section of the journal

Frontiers in Public Health

Received: 19 August 2020

Accepted: 12 October 2020

Published: 23 November 2020

Citation:

Sreenath $K$, Chaudhry R, Vinayaraj $E V$,

Dey AB, Kabra SK, Thakur B and

Guleria R (2020) Distribution of

Virulence Genes and

Sequence-Based Types Among Legionella pneumophila Isolated From the Water Systems of a Tertiary Care

Hospital in India.

Front. Public Health 8:596463.

doi: 10.3389/fpubh.2020.596463

\section{Distribution of Virulence Genes and Sequence-Based Types Among Legionella pneumophila Isolated From the Water Systems of a Tertiary Care Hospital in India}

\author{
K. Sreenath ${ }^{1}$, Rama Chaudhry ${ }^{1 *}$, E. V. Vinayaraj ${ }^{1}$, A. B. Dey ${ }^{2}$, S. K. Kabra ${ }^{3}$, \\ Bhaskar Thakur ${ }^{4 \dagger}$ and Randeep Guleria ${ }^{5}$ \\ ${ }^{1}$ Department of Microbiology, All India Institute of Medical Sciences, New Delhi, India, ${ }^{2}$ Geriatric Medicine, All India Institute \\ of Medical Sciences, New Delhi, India, ${ }^{3}$ Pediatrics, All India Institute of Medical Sciences, New Delhi, India, ${ }^{4}$ Biostatistics, All \\ India Institute of Medical Sciences, New Delhi, India, ${ }^{5}$ Pulmonary, Critical Care, and Sleep Medicine, All India Institute of \\ Medical Sciences, New Delhi, India
}

Background: Legionnaires' disease (LD) is a potentially fatal pneumonia predominantly caused by infection due to Legionella pneumophila although more than 50 other Legionella species are described. Water systems contaminated with Legionella spp. are the implicated sources of Legionnaires' disease. In this study, we aimed to assess Legionella contamination in the water sources of a tertiary care hospital and to determine the virulence properties and molecular characteristics of $L$. pneumophila environmental isolates.

Methods: During May 2015 through August 2018, a total of 201 hospital water samples were tested for L. pneumophila by standardized culture procedures; environmental isolates were examined for the presence of two virulence genes: Legionella vir homolog (Ivh) and repeats in structural toxin ( $r$ txA ) by PCR. The genotyping of isolates was performed by sequence-based typing (SBT) according to the protocol of the European Study Group for Legionella Infections (ESGLI).

Results: L. pneumophila was isolated from 38/201 (18.9\%) water samples; among the 46 isolates, the Ivh locus was present in 45 (97.8\%), the rtxA locus was found in 45 (97.8\%), and both loci were found in $44(95.7 \%)$ isolates. A total of 23 sequence types (STs) were identified among the 44 isolates (index of discrimination [IOD] of 0.929), and $11 / 23(47.8 \%)$ STs were new to the ESGLI database.

Conclusions: The study results showed genetic diversity in L. pneumophila isolates from the hospital environment along with a high percentage of pathogenicity loci. Besides, certain STs may have an increased ability to cause legionellosis, thus requires specific infection control and prevention strategies whenever identified.

Keywords: Legionella, legionellosis, environmental surveillance, virulence, genotyping, sequence-based typing, molecular epidemiology 


\section{INTRODUCTION}

Legionella pneumophila, the etiological agent of atypical pneumonia known as Legionnaires' disease (LD), is the inhabitants of both natural and human-made aquatic environments (1). LD is the most common waterborne disease, and the reported cases of legionellosis have shown rising trends both in the United States and Europe $(2,3)$. Presently, the genus Legionella includes more than 70 distinct serogroups from $>60$ known species, and of these, at least 30 species have been associated with opportunistic infections in humans. $L$. pneumophila accounts for $\sim 90 \%$ of LD cases, and the majority of clinical cases are attributed to serogroup 1 of L. pneumophila (Lp1) $(1,4)$.

Legionella spp. can survive for prolonged periods in aquatic systems, can evade and multiply in free-living protozoa and replicate in the presence of biocides, including chlorine (5). Legionella contamination has been increasingly reported in cooling towers (CT), hot springs, foot spas, drinking and nondrinking water systems of hotels, nursing homes, and health care facilities $(6,7)$. The periodic monitoring of Legionella in the hospital water systems allows for risk prediction and the elimination of this pathogen from possible infection sources (7). In India, LD has been sporadically reported from specific locations, but the disease clusters and outbreaks are so far not identified $(8,9)$. The water safety regulations for Legionella monitoring and decontamination are absent in this country, and no active surveillance program exists for monitoring $L$. pneumophila in the hospital environment.

Studies indicate that difference exists between L. pneumophila strains, particularly in their ability to withstand in external environments and to produce infections in humans. These differences are mainly attributed to the presence or absence of specific genes encoding virulence among bacterial isolates (10). Several virulence genes in Legionella spp. including the type IV secretion system genes, intracellular multiplication/defective in organelle trafficking (icm/dot), tra1, Legionella vir homolog $(l v h)$, type IV pilus genes pilDE, macrophage infectivity potentiator (mip), repeats in structural toxin $(r t x A)$, and enhC have been characterized and are extensively studied (10-12). The $l v h$ locus derives protein for a second type IV secretion system that contributes to conjugation and virulence (13). The $r t x A$ gene is a pore-forming toxin that is regulated by the dot/icm complex that contributes to cellular entry and subsequent adherence to the host cell (14). Previous studies have reported that the $l v h$ and $r t x A$ regions are found more often in strains associated with human disease (10-12). Therefore, in the present study, we aimed to assess Legionella contamination in the water systems of a hospital, to identify the Legionella species and serogroups involved, and to survey the presence of two pathogenicity loci (the lvh and $r t x A$ ) in environmental isolates to determine their infection potential.

Molecular typing of L. pneumophila isolates is foremost important for epidemiological investigation of LD cases, clusters, and outbreaks. An outbreak source can be determined by linking strains from the environment to clinical strains by using different molecular typing methods (15). L. pneumophila isolates can be genotyped by sequence-based typing (SBT) using seven loci including five virulence genes (flaA, pilE, mip, mompS, and proA) and two housekeeping genes (asd and neuA) as proposed by the European Study Group for Legionella Infections (ESGLI) (1618). The SBT is a rapid, reproducible, and highly discriminatory typing technique and, therefore, widely accepted as a gold standard for LD outbreak investigations and rapid identification of isolates that are closely related $(4,16,17)$. Previous typing studies on L. pneumophila isolates indicate the dominance of certain sequence types (STs) in sporadic cases and outbreaks (4). However, from India, so far, no studies were reported to determine the DNA SBT of $L$. pneumophila isolates. Therefore, we further characterized L. pneumophila environmental isolates by SBT analysis and compared our data with the global database available at the ESGLI.

\section{MATERIALS AND METHODS}

\section{Environmental Surveillance}

The study was conducted in a major tertiary care hospital in New Delhi, India, that has organ transplantation and cancer treatment facilities. During May 2015 through August 2018, 21 sites inside the hospital campus that spread over $\sim 115$ acres, including hospital, residential, and general areas, were monitored for the presence of L. pneumophila. Samplings were performed in different buildings hosting patient rooms, intensive care units, clinics, laboratories, and nursing stations. Both potable (PW; drinking water for patients, hospital staff, and public) and non-potable water samples (NPW; bathwater and water for handwashing) were collected, and Legionella testing was carried out four times per year. The samples were collected from distal outlets of hot and cold water taps and AC cooling towers (basin beneath the tower). The water temperature was measured at the time of sample collection by using a precision thermometer (Zeal, England). Legionella isolation and identification from water samples was done following the guidelines issued by the US Centers for Disease Control and Prevention (19). The detailed methodology regarding $L$. pneumophila environmental surveillance has been published elsewhere $(20,21)$.

\section{Legionella Speciation and Identification of L. pneumophila Serogroup 1}

All Legionella isolates collected during the surveillance period were subjected to a real-time PCR assay targeting the ssrA gene for the confirmation of the Legionella genus (22). Further identification of L. pneumophila was done by using a real-time PCR targeting the mip gene, and finally, the detection of Lp1 was done by using another real-time PCR targeting wzm gene (22). DNA isolated from L. pneumophila serogroup 1 ATCC 33152 (Strain Philadelphia) was used as a positive control for the standardization of real-time PCR.

\section{Identification of Virulence Genes by Using PCR}

All L. pneumophila isolates recovered from hospital water samples were surveyed for the presence of two virulence gene loci using previously published primers and PCR conditions $(10,11)$. 
Briefly, six-primer pairs were used in this study including lvh1/prpA-lvh2/prpA, lvh3/lvhB3-lvh4/lvhB4, lvh5/lvhB8lvh6/lvhB9, and lvr1/lvrE-lvr2/lvrE for the amplification of the lwh region and $\mathrm{rtx} 1 / r t x A-\mathrm{rtx} 2 / r t x A$ and $\mathrm{rtx} 3 / r t x A-\mathrm{rtx} 4 / r t x A$ for the identification of the $r t x A$ region. DNA extraction was performed by emulsifying $2-3$ colonies in sterile water and boiling at $100^{\circ} \mathrm{C}$ for $10 \mathrm{~min}$. PCR amplification involved 35 cycles of $1 \mathrm{~min}$ at $95^{\circ} \mathrm{C}$ for denaturation, $1 \mathrm{~min}$ at $55^{\circ} \mathrm{C}$ for annealing, and $1 \mathrm{~min}$ at $72^{\circ} \mathrm{C}$ for the extension. DNA isolated from $L$. pneumophila serogroup 1 ATCC 33152 (Strain Philadelphia) was used as a positive control for PCR.

\section{L. pneumophila Genotyping by Sequence-Based Typing (SBT)}

L. pneumophila SBT was performed by using the seven-gene (flaA, pile, asd, mip, mompS, proA, neuA) protocol SBT scheme according to the guidelines issued by the ESGLI (version 5.0) (16-18). PCR amplification involved 35 cycles of $30 \mathrm{~s}$ at $94^{\circ} \mathrm{C}$ for denaturation, $30 \mathrm{~s}$ at $55^{\circ} \mathrm{C}$ for annealing, and $45 \mathrm{~s}$ at $72^{\circ} \mathrm{C}$ for the extension. After purification and sequencing (Barcode Biosciences Pvt. Ltd., Bangalore, India; Dr. KPC Life Sciences Pvt. Ltd., Kolkata, India), the forward and reverse sequence trace files were uploaded to the online Legionella Sequence Quality Tool (www.hpa-bioinformatics.org.uk/cgi-bin/legionella/ sbt/seq_assemble_legionella1.cgi). Sequence alignment and trimming was performed by the tool and individual alleles, allelic profile, and a sequence type (ST) were identified. For each isolate, the profile of seven alleles at each of the loci was defined in the following order: flaA, pile, asd, mip, mompS, proA, and neuA (e.g., 1, 4, 3, 1, 1, 1, 1). Finally, the ST was indicated by a number (e.g., ST1). For L. pneumophila non-Lp1 strains, if neuA is not amplified with standard neuA primers, amplification was done by using primers targeting neuAh (N-Acylneuraminate Cytidyltransferase homolog) according to an alternative ESGLI protocol (version 1.0) (23). The homolog (neuAh) has been described by Farhat et al. and is reported to be found in certain non-serogroup 1 (non-Lp1) strains when the neuA gene is not amplified with the standard neuA primers in the SBT protocol (23). Therefore, whenever the neuA gene is not amplified, in place of neuA result, the neuAh allele result was used according to the predetermined SBT order (flaA, pilE, asd, mip, mompS, proA, and neuAh) as recommended by the ESGLI (version 1.0). Finally, the combination of alleles is defined as 7-digit allelic profile (e.g., $8,6,34,9,2,8,209$ ) and a ST was represented by a number (e.g., ST1417). Newly identified alleles and STs were submitted to the ESGLI SBT database (http://www.hpa-bioinformatics.org.uk/ legionella/legionella_sbt/php/sbt_homepage.php).

\section{Phylogenetic Analysis}

We assessed the relationships between STs and within clonal complexes by using the goeBURST implemented in the PHYLOViZ program (http://www.phyloviz.net/goeburst/\# Software). The default setting in the eBURST (the stringent group definition) was used; by this definition, a clonal complex contains STs that share six of the seven evaluated SBT alleles with at least one other member of the group and are all believed to be descended from the same founding genotype (primary
TABLE 1 | Legionella pneumophila positivity according to water sampling sites and buildings in a Tertiary care hospital, India, 2015-2018.

\begin{tabular}{|c|c|c|c|c|c|}
\hline $\begin{array}{l}\text { Site } \\
\text { ID }\end{array}$ & $\begin{array}{l}\text { Hospital buildings } \\
\text { located in the site }\end{array}$ & $\begin{array}{l}\text { Sampling } \\
\text { events }\end{array}$ & $\begin{array}{l}\text { L. pneumophila } \\
\text { positive } \\
\text { instances }\end{array}$ & Lp1 & Lp 2-14 \\
\hline 1 & $\begin{array}{l}\text { Outpatient department } \\
\text { (OPD) }\end{array}$ & 6 & 1 & 1 & - \\
\hline 2 & Emergency & 4 & - & - & - \\
\hline 3 & $\begin{array}{l}\text { Hospital wards (floor } \\
1-4 \text { ) }\end{array}$ & 6 & - & - & - \\
\hline 4 & $\begin{array}{l}\text { Hospital wards (floor } \\
5-8 \text { ) }\end{array}$ & 5 & - & - & - \\
\hline 5 & Ophthalmology & 9 & 2 & 2 & - \\
\hline 6 & Oncology & 12 & 1 & 1 & - \\
\hline 7 & $\begin{array}{l}\text { Cardiothoracic and } \\
\text { neurology }\end{array}$ & 11 & 3 & 1 & 2 \\
\hline 8 & $\begin{array}{l}\text { Pulmonology and new } \\
\text { private ward }\end{array}$ & 10 & 3 & 3 & - \\
\hline 9 & $\begin{array}{l}\text { Nursing college, genetic } \\
\text { lab, community } \\
\text { medicine }\end{array}$ & 15 & 6 & 5 & 1 \\
\hline 10 & Dentistry & 5 & - & - & - \\
\hline 11 & $\begin{array}{l}\text { AC cooling tower for } \\
\text { OPD building }\end{array}$ & 7 & 4 & 3 & 1 \\
\hline 12 & Teaching divisions & 15 & 2 & 2 & - \\
\hline 13 & $\begin{array}{l}\text { General areas near } \\
\text { teaching block and } \\
\text { library }\end{array}$ & 5 & - & - & - \\
\hline 14 & Biotechnology & 10 & 1 & 1 & - \\
\hline 15 & $\begin{array}{l}\text { AC cooling towers for } \\
\text { oncology, cardiology, } \\
\text { and neurology buildings }\end{array}$ & 26 & 4 & 2 & 2 \\
\hline 16 & Girls hostels & 7 & 1 & 1 & - \\
\hline 17 & Boys hostels & 11 & 3 & 2 & 1 \\
\hline 18 & Swimming pool & 2 & - & - & - \\
\hline 19 & $\begin{array}{l}\text { Guest house and staff } \\
\text { houses }\end{array}$ & 15 & 5 & - & 5 \\
\hline 20 & $\begin{array}{l}\text { New Resident doctors } \\
\text { hostels }\end{array}$ & 11 & 1 & - & 1 \\
\hline 21 & $\begin{array}{l}\text { Other general areas and } \\
\text { laboratories }\end{array}$ & 9 & 1 & 1 & - \\
\hline
\end{tabular}

Lp, Legionella pneumophila.

founder) (24). Comparative goeBURST analysis was used to relate L. pneumophila STs identified in this study with those reported from Japan, China, and South Korea. The SBT data of 164 isolates from China, 135 isolates from Japan, and 104 isolates from South Korea were obtained from previous studies $(6,25,26)$.

\section{Statistical Analysis}

Categorical variables were expressed in terms of numbers and percentages. The association between characteristics of water samples and L. pneumophila positivity was evaluated through odds ratio (OR) with 95\% CI (confidence interval). A $p$ value (two-tailed) below 0.05 was considered to be statistically significant. The analysis was performed by using function 
TABLE 2 | Legionella pneumophila isolated from 201 water samples collected at a tertiary healthcare center, by type of water, sampling site, temperature, year and period, India, May 2015- August 2018.

\begin{tabular}{|c|c|c|c|c|c|c|}
\hline S.no & Characteristic & $\begin{array}{l}\text { Total no of } \\
\text { samples } \\
\text { collected } \\
(n=201)\end{array}$ & $\begin{array}{c}\text { Lp positive } \\
(n=38)[n(\%)]\end{array}$ & $\begin{array}{c}\text { Lp negative } \\
(n=163)[n(\%)]\end{array}$ & OR $95 \% \mathrm{Cl}$ & $p$-value \\
\hline \multirow[t]{3}{*}{1} & Type of water samples & & & & & \\
\hline & a. Potable & 113 & $19(16.8)$ & $94(83.2)$ & 1.00 & \\
\hline & b. Non-potable & 88 & $19(21.5)$ & $69(78.4)$ & $1.36(0.63-2.94)$ & 0.391 \\
\hline \multirow[t]{5}{*}{2} & Sampling site & & & & & \\
\hline & a. Patient areas & 68 & $10(14.7)$ & $58(85.3)$ & 1.00 & \\
\hline & b. Residential areas & 44 & $10(22.7)$ & $34(77.3)$ & $1.71(0.57-5.01)$ & 0.279 \\
\hline & c. Cooling towers & 33 & $8(24.2)$ & $25(75.8)$ & $1.86(0.56-5.91)$ & 0.240 \\
\hline & d. Other areas & 56 & $10(17.9)$ & $46(82.1)$ & $1.26(0.43-3.69)$ & 0.635 \\
\hline \multirow[t]{5}{*}{3} & Temperature range & & & & & \\
\hline & a. $<20^{\circ} \mathrm{C}$ & 39 & $8(20.5)$ & $31(79.5)$ & 1.00 & \\
\hline & b. $20-29^{\circ} \mathrm{C}$ & 118 & $18(15.3)$ & $100(84.7)$ & $0.70(0.26-2.04)$ & 0.444 \\
\hline & c. $30-39^{\circ} \mathrm{C}$ & 40 & $11(27.5)$ & $29(72.5)$ & $1.47(0.46-4.84)$ & 0.467 \\
\hline & $\mathrm{d}>40^{\circ} \mathrm{C}$ & 4 & $1(25)$ & $3(75)$ & 1.29 (0.02-18.69) & 0.834 \\
\hline \multirow[t]{6}{*}{4} & Seasonality & & & & & \\
\hline & a. Winter (Dec-Jan) & 20 & $4(20)$ & $16(80)$ & 1.00 & \\
\hline & b. Spring (Feb-March) & 23 & $3(13)$ & $20(86.9)$ & $0.60(0.08-4.17)$ & 0.538 \\
\hline & c. Summer (April-June) & 60 & $13(21.7)$ & 47 (78.3) & $1.11(0.28-5.32)$ & 0.875 \\
\hline & d. Monsoon (July- August) & 44 & $8(18.2)$ & $36(81.8)$ & $0.89(0.20-4.64)$ & 0.863 \\
\hline & e. Autumn (Sept-Nov) & 54 & $10(18.5)$ & $44(81.5)$ & $0.91(0.22-4.54)$ & 0.885 \\
\hline \multirow[t]{3}{*}{5} & Periodicity & & & & & \\
\hline & $\begin{array}{l}\text { a. First period } \\
\text { (Feb, 2015-Jan, 2017) }\end{array}$ & 79 & $21(26.6)$ & $58(73.4)$ & 1.00 & \\
\hline & $\begin{array}{l}\text { b. Second period } \\
\text { (Feb, 2017-Sept, 2018) }\end{array}$ & 122 & 17 (13.9) & $105(86.1)$ & $0.45(0.20-0.97)$ & 0.030 \\
\hline \multirow[t]{3}{*}{6} & Age of building & & & & & \\
\hline & a. $>10$ years & 187 & $36(19.3)$ & $151(80.7)$ & 1.00 & \\
\hline & b. $<10$ years & 14 & $2(14.3)$ & $12(85.7)$ & $0.70(0.07-3.36)$ & 0.647 \\
\hline
\end{tabular}

Cl, confidence interval; Lp, Legionella pneumophila; OR, odds ratio. Bold text indicates statistical significance.

odds. ratio in R-version 3.6.1. The index of diversity (IOD) was determined using Hunter and Gaston's modification of Simpson's index of diversity according to a previously described method (27).

\section{RESULTS}

\section{Environmental Surveillance}

Among the 201 water samples collected during the study period, 38 (18.9\%; 19 potable and 19 non-potable) were positive for L. pneumophila by culture. Of these, 10 (26.3\%) each sample was collected from patient areas, residential areas, and general hospital areas, and $8(21 \%)$ were from AC cooling towers. Regarding the 38 samples tested positive for L. pneumophila, the presence of Lp1 was detected in 25 (65.8\%) samples, which included 11 potable and 14 non-potable water samples. The remaining $13(34.2 \%)$ samples were contaminated due to Lp 2-14 serogroups. The temperature of L. pneumophila positive water samples ranged from 12 to $57^{\circ} \mathrm{C}$ (median temperature of $25^{\circ} \mathrm{C}$ ), and the pathogen was most frequently isolated from a temperature range of $20^{\circ} \mathrm{C}-40^{\circ} \mathrm{C}$ (during 29 instances).
Among the 21 sampling sites, 15 were positive for $L$. pneumophila during at least one sampling event, two sites were positive for Legionella during two instances, three sites were positive during three instances, and four sites were positive during $>4$ instances. These four sites (two drinking water units and two AC cooling towers) that repeatedly tested positive for L. pneumophila were identified as high-risk sites. L. pneumophila positivity for the sampling sites and buildings located within these sites are shown in Table $\mathbf{1 .}$

We compared water samples with and without L. pneumophila and assessed characteristics including type, source, and temperature range of water samples, and age of water tanks or storage systems. None of these factors were found to have a significant association with L. pneumophila positivity (Table 2). During the study period, seasonal variations in Legionella positivity were not observed; the contamination was found to be consistent throughout the year.

\section{Legionella Speciation and Identification of L. pneumophila Serogroup 1}

A total of 47 L. pneumophila isolates were obtained from all positive samples during the study period. The number 


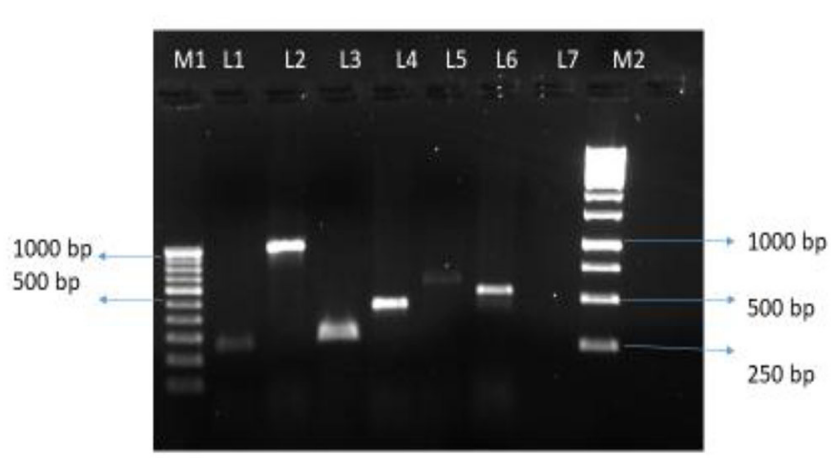

FIGURE 1 | Legionella pneumophila isolate from water tested positive for six pairs of primers. Lane M1: 100 bp DNA ladder (100-1,000 bp) with DNA sizes indicated. Lane 1 to 6 , PCR products for primer pairs for the IVh region: Ivh1/prpA-Ivh2/prpA (L1, 259 bp PCR product), Ivh3/IvhB3-Ivh4/IvhB4 (L2, 1007 bp PCR product), Ivh5/IvhB8- Ivh6//vhB9 (L3, 294 bp PCR product), and Ivr1//vrE-Ivr2/IvrE ( $L 4,423$ bp PCR product), and for the $r$ txA region:

rtx1/rtxA-rtx2/rtxA (L5, 603 bp PCR product), and rtx3/rtxA-rtx4/rtxA (L6, 543 bp PCR product), Lane 7, blank, Lane M2: $1 \mathrm{~Kb}$ DNA ladder with representative sizes of DNA are indicated.

of Legionella isolates is not equal to the number of positive samples because, during some instances, we isolated different L. pneumophila serogroups (both Lp1 and Lp 2-14 [non-Lp1]) in the same sample. All isolates $(n=47)$ were identified as $L$. pneumophila by real-time PCR targeting the mip gene. Twentynine out of 47 (61.7\%) isolates were identified as Lp1 by $w z m$ gene real-time PCR, and the remaining isolates $(n=18$ [38.3\%]) were referred to as Lp 2-14. Serogrouping for the non-Lp1 isolates was not performed.

\section{Identification of Virulence Genes by Using PCR}

Of 47 L. pneumophila isolates, 46 were subjected to the identification of virulence genes. For one isolate, DNA was found to be degraded; therefore, excluded from the analysis. Among the tested isolates, at least one virulence gene loci ( $l v h$ or $r t x A$ ) was detected in all (100\%) isolates. Specifically, the $l v h$ locus was present in $45(97.8 \%)$ isolates, the $r t x A$ locus was found in 45 (97.8\%), and both loci were found in $44(95.7 \%)$ isolates. The simultaneous absence of the two loci was not observed in any of the tested isolates. Among the two groups (Lp1 and nonLp1), both the gene loci were present in all Lp1 $(n=29,100 \%)$ isolates tested. Of the non-Lp1 $(n=17)$ isolates, 15 (88.3\%) tested positive for both genes, and the remaining two isolates showed the following pattern: $l v h$-positive, $r t x A$-negative and $l v h$ negative, $r t x A$-positive. Figure 1 shows PCR positive products in an environmental isolate tested for all primer pairs. Test results of the detection of virulence genes are shown in Table 3.

\section{L. pneumophila Genotyping by Sequence-Based Typing (SBT)}

SBT analysis assigned 44 environmental isolates into 23 distinct STs (IOD, 0.929). DNA of three isolates was found to be degraded;
TABLE 3 | Detection of virulence genes in Legionella pneumophila isolates from water samples.

\begin{tabular}{lllll}
\hline Legionella spp. & $\begin{array}{l}\text { No of } \\
\text { isolates } \\
\text { tested }\end{array}$ & $\begin{array}{l}\text { Ivh locus } \\
\mathbf{n}(\%)\end{array}$ & $\begin{array}{l}\boldsymbol{r t x A} \text { locus } \\
\mathbf{n}(\%)\end{array}$ & $\begin{array}{l}\text { Ivh and } \boldsymbol{r t x A} \\
\text { loci } \mathbf{n}(\%)\end{array}$ \\
\hline $\begin{array}{l}\text { L. pneumophila serogroup } \\
\text { 1(Lp1) }\end{array}$ & 29 & $29(100)$ & $29(100)$ & $29(100)$ \\
$\begin{array}{l}\text { L. pneumophila serogroup } \\
\text { 2-14 (non-Lp1) }\end{array}$ & 17 & $16(94.1)$ & $16(94.1)$ & $15(88.2)$ \\
\hline Total & 46 & $45(97.8)$ & $45(97.8)$ & $44(95.6)$ \\
\hline
\end{tabular}

hence genotyping was not applied. For one Lp1 isolate, ST was not able to determine (Table 4). Six STs were associated with more than one isolate, and 17 STs were identified with one single isolate. The most predominant ST in this study was ST1, with $22.7 \%(10 / 44)$ of the isolates belonging to this sequence type. The other STs obtained more than once among the tested isolates were ST763 $(n=4 ; 9.1 \%)$, ST2848 $(n=4 ; 9.1 \%)$, ST2854 $(n=3 ; 6.8 \%)$, ST2868 $(n=3 ; 6.8 \%)$, and ST114 $(n=2 ; 4.5 \%)$. We identified the presence of the neuAh allele in 8 non-Lp1 isolates, which were not amplified using the standard neuA primers. They contained five different neuAh alleles, including neuAh 201, 207, 208, 209, and 228 (Table 4).

Querying the ESGLI database (available at http://www.ewgli. org), it was found that $11 / 23(47.8 \%)$ STs were identified for the first time in this study. They are ST2848, ST2849, ST2850, ST2854, ST2855, ST2865, ST2866, ST2867, ST2868, ST2869, and ST2874. We also identified one new allele of the mip gene (mip 93) (Table 4).

Strains with indigenous STs were isolated from different water sources. Nine cooling tower (CT) isolates were divided into 6 STs (IOD, 0.888), 24 PW isolates were divided into 14 STs (IOD, 0.920), and $11 \mathrm{NPW}$ isolates were divided into 8 STs (IOD, 0.933; except one isolate of which ST was not determined). ST1 was found in all three sources (PW; $n=6, \mathrm{NPW} ; n=3$, and CT; $n=1)$. Other common STs found across different water environments included ST2854 (CT; $n=2$, NPW; $n=1)$, ST2848 (CT; $n=3$, PW; $n=1$ ), and ST763 (PW; $n=3$, CT; $n=1$ ). The only ST common across the patient, residential, and general areas of this hospital was ST1. Apart from ST1, 5 STs including ST114, ST322, ST1095, ST2849 (a new ST), and ST2869 (a new ST) were found to be present in the patient areas. Twenty-seven isolates of Lp1 were assigned into 11 STs (IOD, 0.824; except one isolate of which ST was not determined), and 17 non-LP1 isolates were assigned into 14 STs (IOD, 0.955). The common ST appeared in both groups (Lp1 and non-Lp1) was only ST114.

\section{goeBURST Analysis}

We applied goeBURST analysis to examine the relationship between STs obtained in this study with a single-locus variant selected (SLV). Five STs from this study were predicted to form two clonal complexes, whereas the remaining 18 STs did not relate to each other, therefore, identified as singletons. Clonal 
TABLE 4 | Result of molecular typing of $L$. pneumophila isolates by sequence-based typing (SBT).

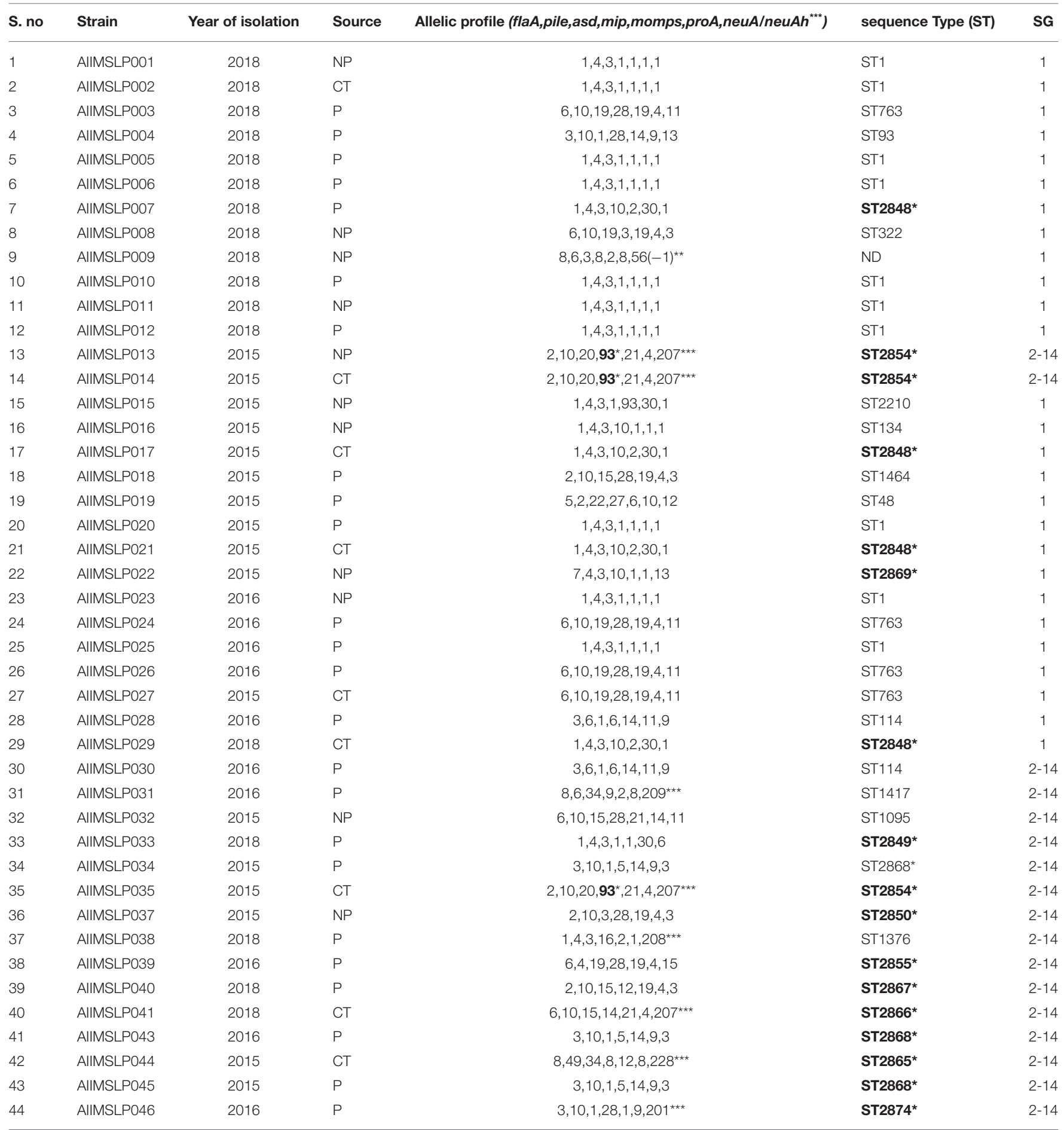

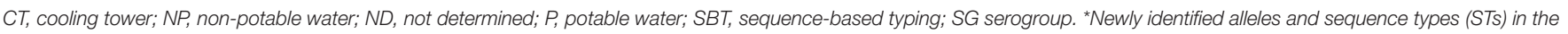

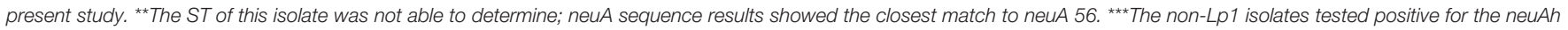
allele. The neuAh nomenclature starts from 201, to differentiate between the neuA and neuAh alleles. Bold text indicates newly identified allele and STs in this study.

complex 1 (CC-1) consisted of 3 STs (ST1464, ST2850, ST2867), and $3 / 44(6.8 \%)$ isolates belonged to this complex. The CC-2 consisted of two STs (ST1 and ST134), and 11/44 (25\%) isolates belonged to this complex. The phylogenetic relationship between L. pneumophila STs identified by goeBURST analysis is shown in Figure 2. 


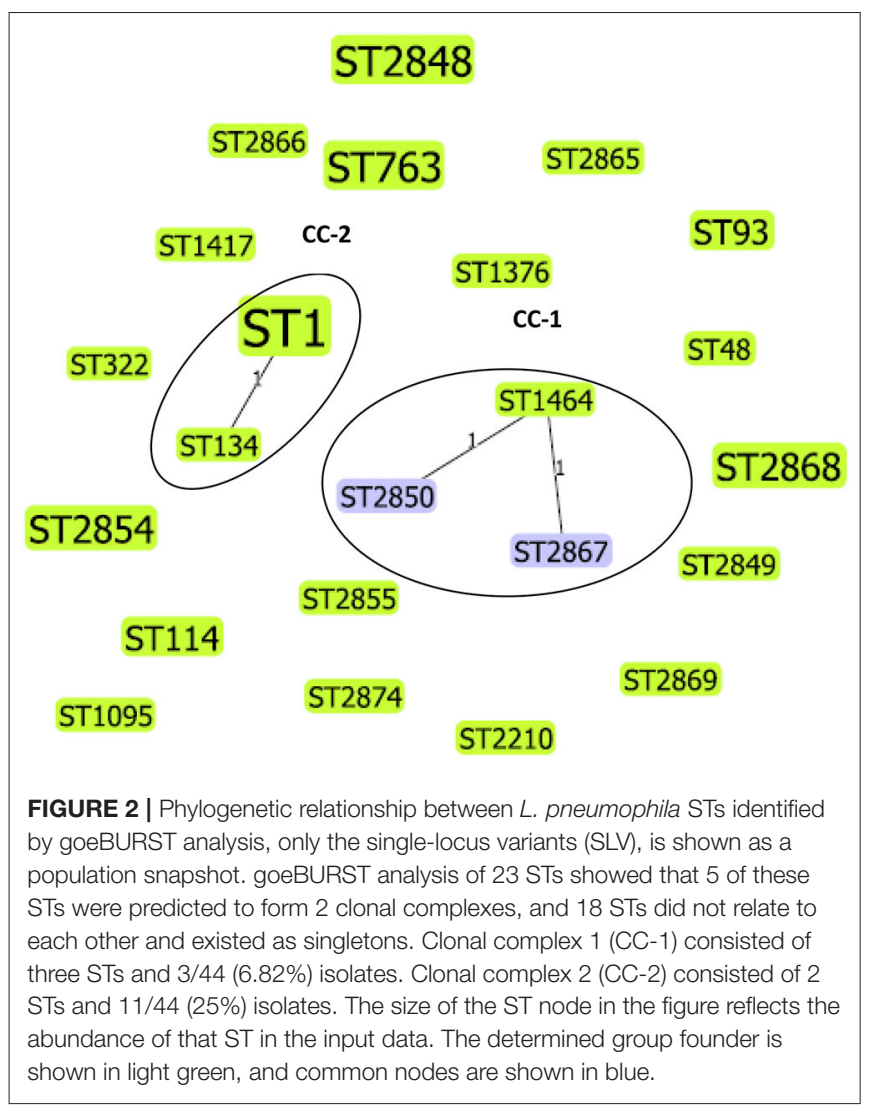

\section{Comparative goeBURST Analysis of L. pneumophila Environmental Isolates From India, China, Japan, and South Korea}

Forty-four L. pneumophila isolates detected in the present study were compared with 403 Lp1 environmental isolates from Japan $(n=135)$, China $(n=164)$, and South Korea $(n=104)$. The SBT data of Chinese, Japanese, and South Korean isolates were obtained from previously published studies $(6,25,26)$. Altogether, these 447 isolates were divided into 127 STs. Most of these STs exclusively belonged to one country, and the only ST that found to be present in all the four countries was ST1. Apart from ST1, ST1464 detected in this study was also reported from China; similarly, ST48 was also reported from Japan. The comparative goeBURST analysis grouped 67 STs into 16 clonal complexes (CCs), and 60 STs were identified as singletons (Figure 3). Among the $16 \mathrm{CCs}$, four clonal complexes (CC-A, CC-C, CC-F, and CC-I) also contained STs identified in this present study.

ST1 was the determined primary founder of the largest clonal complex CC-A; it had 13 SLVs, including ST134, which is detected in the present study. The CC-C with determined primary founder ST278 and subgroup founder ST129 contained ST1095, a ST identified in our study that shares 6/7 alleles with ST278 (ST reported from Japan). The CC-F, which is observed in the present study, contained ST1464 as a predicted primary founder and ST2867 and ST2850 as obtained SLVs. Another ST reported in this study, ST763 belonged to CC-I and is closely related to ST600 (a ST reported from Japan) (Figure 3).

\section{DISCUSSION}

\section{Environmental Surveillance}

Drinking water colonization by Legionella spp. is directly linked to the occurrence of HALD, and several national public health agencies have mandated routine environmental surveillance as a preventive measure. In our study, L. pneumophila was isolated from $18 \%$ of hospital water samples, and these findings are in line with a previous study from India that reported a positivity of 15\% (28). In studies reported from Spain and Italy, the occurrence of Legionella in the hospital environment has been found to be higher (60 and $74.1 \%$, respectively) $(29,30)$. Surveys of Legionella colonization in hospitals have been performed in the USA, UK, Canada, and Spain, with Legionella positivity rates varied from 12 to $85 \%(31,32)$. In a large-scale hospital survey conducted in Taiwan (belonging to East Asia), L. pneumophila contamination was found in the water systems of 10 out of 16 hospitals (33).

In the present study, during 19 instances, potable water tested positive for L. pneumophila, and it is reported that in hospital settings, potable water rather than cooling towers has been implicated as a potential source of legionellosis (7). One possible solution to prevent the spread of Legionella from the water was the application of filters to the taps, thus allowing safe water free of the pathogen (34). Therefore, as an initial infection control measure, point-of-use filters were applied to the drinking water taps from where Legionella was isolated. Repeat sampling from these sites did not show Legionella re-colonization after 6 and 12 months. However, to evaluate the efficacy of any Legionelladisinfection method, monitoring over a prolonged period is required. The cooling towers were cleaned and disinfected at least once every 12 months, and water was sampled and tested at least once every 3 months for Legionella spp. These sites were found to be positive for Legionella after 4 months, and the bacteria were isolated from cooling towers until they closed for the next cycle of annual maintenance. Legionella isolation rates reduced significantly in water systems from an average of $26.6 \%$ during Feb 2015- January 2017 to an average of 13.9\% during February 2017-September 2018 (OR 0.45; 95\% CI 0.20-0.97; $p=$ 0.03). This reduction in Legionella isolation rates could be due to Legionella-specific interventions and control measures.

As a part of Legionella risk management, the physicians were informed regarding Legionella colonization in the hospital water systems, and intensive clinical surveillance for this pathogen was initiated. Furthermore, in the future, if a highlevel Legionella colonization is observed in this facility, it is pertinent to install a systemic disinfection system with long termcommitment with a specific aim of preventing legionellosis in the exposed population.

\section{Detection of Virulence Genes}

The $l v h$ and $r t x A$ loci are seen frequently in L. pneumophila isolates associated with human infections. Therefore, these loci can be used as markers for determining the infection potential of isolates (10-12). Our results showed that these 


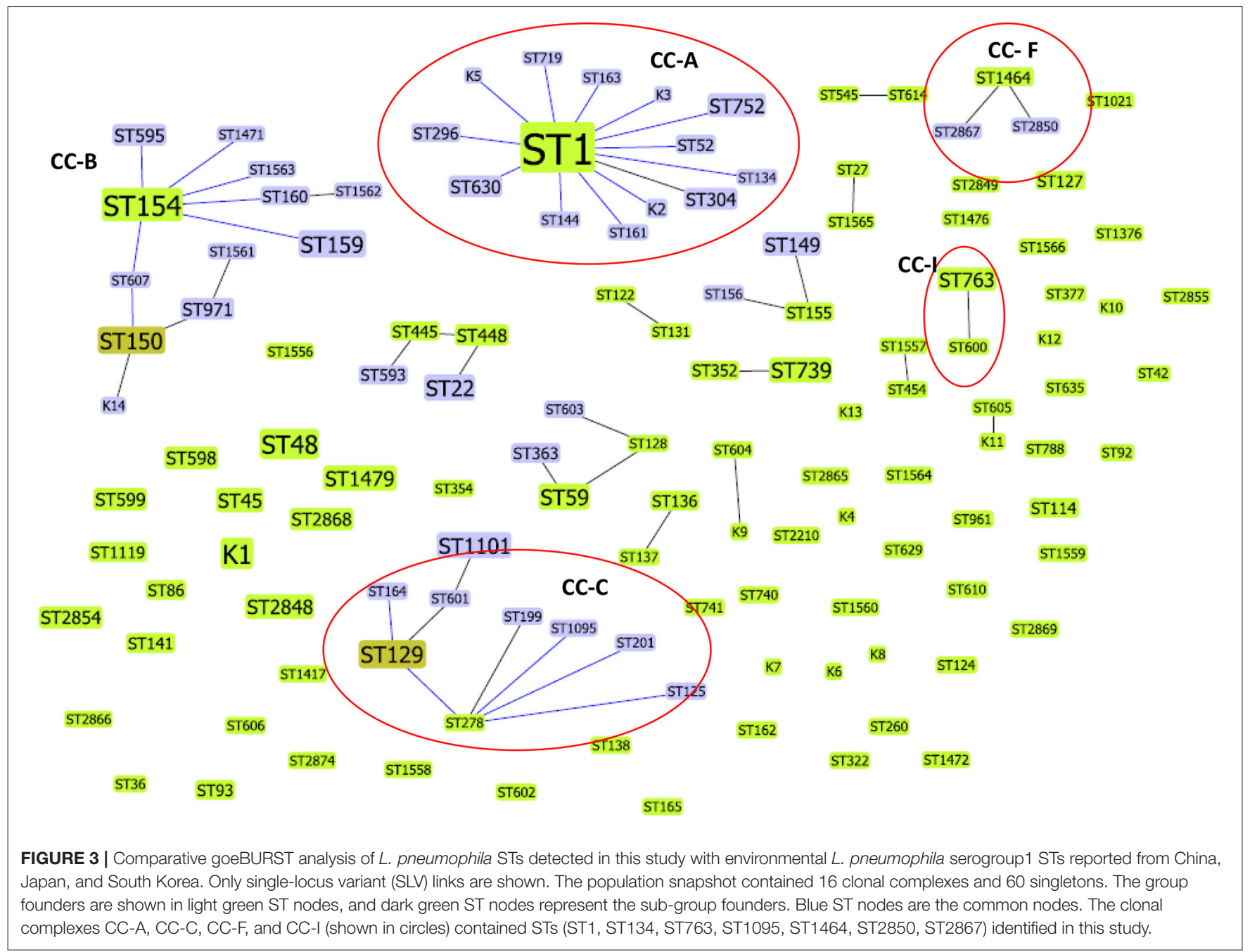

genetic loci are found at a very high percentage in $L$. pneumophila strains from hospital water systems. This finding is in agreement with studies from Greece, Australia, and China that reported a high percentage of the pathogenicity loci in $L$. pneumophila environmental isolates $(11,35,36)$. Despite the high prevalence L. pneumophila containing virulence genes in our hospital environment, HALD clusters were not identified during the clinical surveillance. Similarly, even though Legionella colonization was observed in $24 \%$ of tap waters in Singapore, HALD cases were not identified during 1998-2002 (33, 37). However, in many health care facilities, HALD cases have been discovered after the implementation of Legionella environmental monitoring and clinical surveillance $(7,32)$. Our results indicate the presence of disease-causing L. pneumophila in the hospital environment; therefore, warrant the necessity of investigating Legionella among all patients having nosocomial pneumonia in this facility.

\section{L. pneumophila Sequence-Based Typing}

Over the last decade, SBT analysis has been accepted as a gold standard for the genotyping of L. pneumophila isolates. Besides,
SBT can be applied to study the genetic diversity and clonal expansion of L. pneumophila populations. The method has the advantage of better classification potential, good reproducibility, and is more economical $(4,16-18)$. Our study represents SBT analysis of environmental L. pneumophila isolated from the water systems of a tertiary health care center in India, and the results depict the genetic diversity of this pathogen even though all isolates were derived from the human-made environment. The IOD of the 44 environmental isolates was found to be 0.929 that is higher compared to studies reported from Canada (IOD, 0.888), Japan (IOD, 0.886), and the USA (IOD, 0.751), but lower than those reported from Singapore (IOD, 0.970) $(4,25,38,39)$. We also found that non-Lp1 isolates (IOD, 0.955) are more variable than Lp1 isolates (IOD, 0.824), which could be due to the high prevalence of ST1 among the Lp1 isolates. Additionally, NPW isolates were found to have high IOD (IOD, 0.933) followed by PW (IOD, 0.920) and CT isolates (IOD, 0.888).

ST1, the most common ST distributed throughout the world, was the dominant profile in this study $(4,6,25,26,39)$. Multiple outbreaks due to ST1 strains have been reported from the USA, Canada, and Europe $(4,39)$. ST1 isolates are well-adapted to 
TABLE 5 | The ESGLI database information regarding the L. pneumophila STs identified in this study (as of 11, January, 2020).

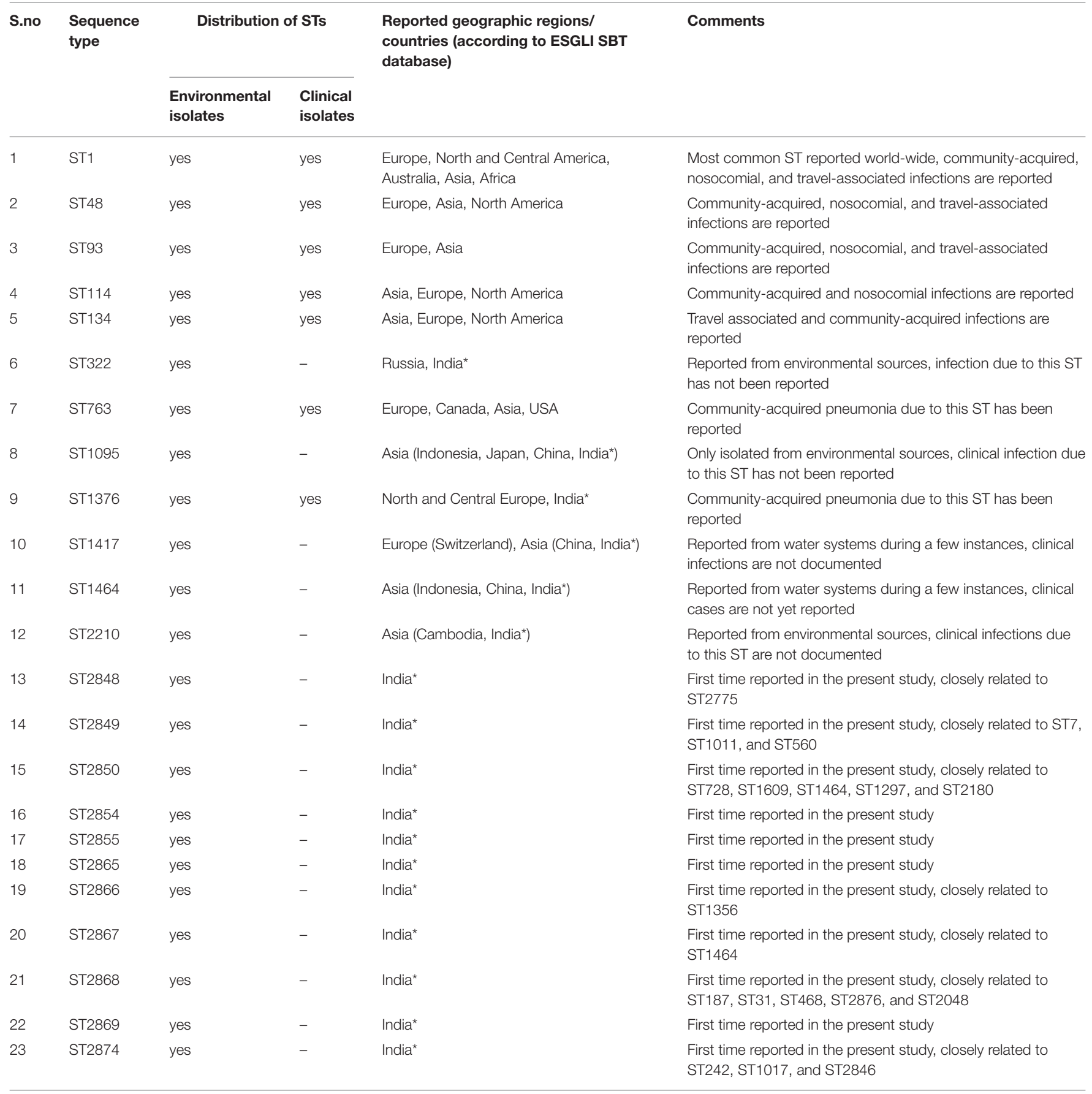

survive in human-made water environments such as a cooling tower, and the ability of this ST to adapt to natural water environments, including hot springs and soil, is found to be low (25). From studies conducted in Japan and South Korea, it was observed that most of the Lp1 environmental isolates, especially those from CT, belonged to ST1 $(25,26)$. In contrast to these findings, in the present study, ST1 was the most dominant ST in Lp1 isolates from PW (42.8\%), followed by those from NPW (33.3\%) and CT (16.6\%). In a Chinese study, ST1 accounted for 92.3 and $53.1 \%$ of the isolates from PW and CT, respectively (6).

Additionally, in a US study, it was reported that ST1 was the dominant ST in both PW and CT isolates (4). These 
differences could be possibly due to the predominance of specific STs or unique strains in various water systems types in these countries. Besides, the genes coding for proteins (e.g., flagellum, pilin, outer membrane protein, macrophage infectivity potentiator, Zinc metalloproteinase) may interact with external environments; therefore, an isolate to get adapt to an environmental source may have a particular ST suitable for each environment (40).

L. pneumophila serogroup 1 STs previously reported from Japan, China, and South Korea (countries belong to East Asia) were compared with STs obtained in this study. The results indicated that some STs (e.g., ST1) are distributed world-wide across different countries, but some clones and STs could be unique circulating only in specific regions. The possibility of recombination events between $L$. pneumophila STs has been previously reported $(6,25)$. ST161 $(11,4,3,1,1,1,1)$ in CC-A was reported to be a recombinant between ST1 $(1,4,3,1,1,1,1)$ and $\operatorname{ST} 154(11,14,16,16,15,13,2)$ the determined primary founders of CC-A and CC-B, respectively (Figure 3). Similarly ST2850 $(2,10,3,28,19,4,3)$ which is identified in our study was a recombinant of ST1464 $(2,10,15,28,19,4,3)$ and ST1 $(1,4,3,1,1,1,1)$ the predicted primary founders of CC-1 and CC-2, respectively (Figure 2 ).

Querying the ESGLI SBT database (available at http://www. esgli.org), it was found that of the 23 STs we obtained in this study, 11 were new to the database, and among the remaining STs, 3 (ST1095, ST1464, ST2210) were unique to Asia. Notably, all three STs were reported to be isolated from environmental sources, and no clinical infections due to these STs have been documented so far (Table 5). It will be interesting to see whether these STs will be associated with any LD cases or outbreaks in the future. The goeBURST analysis has shown that of the 11 STs that are newly identified in this study, two STs have single-locus variants (SLVs), 5 STs have double-locus variants (DLVs), and 2 had triple-locus variants (TLV) within our STs. Besides, querying the ESGLI database, it was found that 7/11 STs are having SLVs abroad (Table 5). Further studies are needed to determine if these STs will persist in this geographical region or expand to other continents.

According to the ESGLI SBT database, few STs described in this study are found to be associated with LD cases and outbreaks. Clinical infections due to STs, including ST1, ST48, ST93, ST114, ST134, ST763, and ST1376, are reported in the ESGLI SBT database (Table 5). These STs that are common to clinical isolates may have an increased ability to cause LD; therefore, their water system sources represent a potential source of legionellosis. ST1 and ST134 (representing CC-2 in this study, Figure 2) were associated with multiple sporadic cases and outbreaks in many parts of the world $(4,39)$. Furthermore, it was hypothesized that similar to certain Legionella spp. and serogroups, few STs also have an enhanced ability to cause infections in humans (41). Hence Legionella control strategies can specifically target these STs that cause the majority of human infections.

During the study period, we could not obtain clinical isolates from patients for genotyping, but SBT was performed directly on the respiratory sample (BAL fluid) of an LD casepatient who was diagnosed by PCR and urine antigen testing (BinaxNOW, Alere, USA). Briefly, we used a nested PCR derived SBT directly to the DNA isolated from respiratory fluid and assigned allele, and finally, a sequence type (ST) using the online ESGLI SBT database (42). We identified that the infection was due to ST1, but was not able to perform epidemiological investigations to determine the possible source of the infection as the patient has acquired infection from another facility. Further studies are needed to analyze the correlation between L. pneumophila environmental and clinical isolates from this region.

Even though we described the distribution and classification of environmental L. pneumophila isolates in a healthcare facility, in future, large-scale studies using whole-genome sequencing (WGS) are needed to classify L. pneumophila environmental and clinical isolates and to identify factors that give fitness to this bacteria to survive in the aquatic environments and to infect humans.

\section{DATA AVAILABILITY STATEMENT}

The original contributions presented in the study are publicly available. This data can be found here: GenBank MT890971.

\section{ETHICS STATEMENT}

The studies involving human participants were reviewed and approved by The ethics committee of AIIMS, New Delhi. The patients/participants provided their written informed consent to participate in this study.

\section{AUTHOR CONTRIBUTIONS}

RC: conceptualization, project administration, resources, validation, writing-review, and editing. $\mathrm{KS}$ and RC: data curation. KS, RC, and BT: formal analysis. KS and RC: investigation and methodology. $\mathrm{KS}$ and $\mathrm{EV}$ : software. $\mathrm{RC}, \mathrm{SK}, \mathrm{AD}, \mathrm{RG}$ : supervision. KS: writing-original draft. All authors contributed to the article and approved the submitted version.

\section{ACKNOWLEDGMENTS}

We thank Dr. Baharak Afshar, Public Health England, London for providing access to the ESGLI SBT database and for assigning the newly identified allele and sequence types. We acknowledge Dr. Arti Kapil, Dr. Bijay Ranjan Mirdha, Dr. Benu Dhawan, and Dr. Urvashi B Singh for their valuable input and suggestions. We also thank Dr. Arvind Valavane for helpful discussions and Dr. Achuthan C Raghavamenon for critical reading of the manuscript. KS acknowledges the Indian Council for Medical Research (ICMR) for providing Senior Research Fellowship (SRF) during the study period. 


\section{REFERENCES}

1. Fields BS, Benson RF, Besser RE. Legionella and legionnaires' disease: 25 years of investigation. Clin Microbiol Rev. (2002) 15:506-26. doi: 10.1128/CMR.15.3.506-526.2002

2. Centers for Disease Control and Prevention. Legionnaires' Disease Surveillance Summary Report, 2014-2015 (2008).

3. European Centre for Disease Prevention and Control. Legionnaires' Disease in Europe, 2015. Stockholm: ECDC (2017).

4. Kozak-Muiznieks NA, Lucas CE, Brown E, Pondo T, Taylor TH, Frace M, et al. Prevalence of sequence types among clinical and environmental isolates of Legionella pneumophila serogroup 1 in the United States from 1982 to 2012. J Clin Microbiol. (2014) 52:201-11. doi: 10.1128/JCM.01973-13

5. Harb OS, Gao LY, Kwaik YA. From protozoa to mammalian cells: a new paradigm in the life cycle of intracellular bacterial pathogens. Environ Microbiol. (2000) 2:251-65. doi: 10.1046/j.1462-2920.2000.00112.x

6. Qin T, Zhou H, Ren H, Guan H, Li M, Zhu B, et al. Distribution of sequencebased types of Legionella pneumophila serogroup 1 strains isolated from cooling towers, hot springs, and potable water systems in China. Appl Environ Microbiol. (2014) 80:2150-7. doi: 10.1128/AEM.03844-13

7. Sabria M, Victor LY. Hospital-acquired legionellosis: solutions for a preventable infection. Lancet Infect Dis. (2002) 2:368-73. doi: 10.1016/S1473-3099(02)00291-8

8. Chaudhry R, Valavane A, Sreenath K, Choudhary M, Sagar T, Shende T, et al. Detection of Mycoplasma pneumoniae and Legionella pneumophila in patients having community-acquired pneumonia: a multicentric study from New Delhi, India. Am J Trop Med Hyg. (2017) 97:1710-6. doi: 10.4269/ajtmh.17-0249

9. Chaudhry R, Sreenath K, Agrawal SK, Valavane A. Legionella and legionnaires' disease: time to explore in India. Indian J Med Microbiol. (2018) 36:324. doi: 10.4103/ijmm.IJMM_18_298

10. Samrakandi MM, Cirillo SL, Ridenour DA, Bermudez LE, Cirillo JD. Genetic and phenotypic differences between Legionella pneumophila strains. J Clin Microbiol. (2002) 40:1352-62. doi: 10.1128/JCM.40.4.1352-1362.2002

11. Huang B, Heron BA, Gray BR, Eglezos S, Bates JR, Savill J. A predominant and virulent Legionella pneumophila serogroup 1 strain detected in isolates from patients and water in Queensland, Australia, by an amplified fragment length polymorphism protocol and virulence gene-based PCR assays. J Clin Microbiol. (2004) 42:4164-8. doi: 10.1128/JCM.42.9.4164-4168.2004

12. Huang B, Yuan Z, Heron BA, Gray BR, Eglezos S, Bates JR, et al. Distribution of 19 major virulence genes in Legionella pneumophila serogroup 1 isolates from patients and water in Queensland, Australia. J Med Microbiol. (2006) 55:993-7. doi: 10.1099/jmm.0.46310-0

13. Segal G, Russo JJ, Shuman HA. Relationships between a new type IV secretion system and the icm/dot virulence system of Legionella pneumophila. Mol Microbiol. (1999) 34:799-809. doi: 10.1046/j.1365-2958.1999.01642.x

14. D’Auria G, Jiménez N, Peris-Bondia F, Pelaz C, Latorre A, Moya A. Virulence factor RTX in Legionella pneumophila, evidence suggesting it is a modular multifunctional protein. BMC Genomics. (2008) 9:14. doi: $10.1186 / 1471-2164-9-14$

15. Fry NK, Alexiou-Daniel S, Bangsborg JM, Bernander S, Pastoris MC, Etienne J, et al. A multicenter evaluation of genotypic methods for the epidemiologic typing of Legionella pneumophila serogroup 1: results of a pan-European study. Clin Microbiol Infect. (1999) 5:462-77. doi: 10.1111/j.1469-0691.1999.tb00176.x

16. Gaia V, Fry NK, Afshar B, Lück PC, Meugnier H, Etienne J, et al. Consensus sequence-based scheme for epidemiological typing of clinical and environmental isolates of Legionella pneumophila. J Clin Microbiol. (2005) 43:2047-52. doi: 10.1128/JCM.43.5.2047-2052.2005

17. Ratzow S, Gaia V, Helbig JH, Fry NK, Lück PC. Addition of neuA, the gene encoding $\mathrm{N}$-acylneuraminate cytidylyl transferase, increases the discriminatory ability of the consensus sequence-based scheme for typing Legionella pneumophila serogroup 1 strains. J Clin Microbiol. (2007) 45:19658. doi: 10.1128/JCM.00261-07

18. Mentasti M, Fry NK. Sequence-Based Typing Protocol for Epidemiological Typing of Legionella pneumophila. ESCMID Study Group for Legionella Infections, European Society of Clinical Microbiology and Infectious Diseases, Basel, Switzerland (2012).
19. Centers for Disease Control and Prevention. Procedures for the Recovery of Legionella From the Environment. Atlanta: US Department of Health and Human Services, Public Health Service (2005). p. 1-13.

20. Chaudhry R, Sreenath K, Arvind V, Vinayaraj EV, Tanu S. Legionella pneumophila serogroup 1 in the water facilities of a tertiary healthcare center, India. Emerg Infect Dis. (2017) 23:1924-5. doi: 10.3201/eid2311.171071

21. Sreenath K, Chaudhry R, Vinayaraj EV, Thakur B. Antibiotic susceptibility of environmental Legionella pneumophila isolated in India. Future Microbiol. (2019) 14:661-9. doi: 10.2217/fmb-2019-0049

22. Benitez AJ, Winchell JM. Clinical application of a multiplex real-time PCR assay for simultaneous detection of legionella species, Legionella pneumophila, and Legionella pneumophila serogroup 1. J Clin Microbiol. (2013) 51:348-51. doi: 10.1128/JCM.02510-12

23. Mentasti M, Fry NK. Analysis of the $N$-acylneuraminate cytidyltransferase homologue (neuAh) found in some non-serogroup 1 strains of Legionella pneumophila, version 1.0. ESCMID Study Group for Legionella Infections, European Society of Clinical Microbiology and Infectious Diseases, Basel, Switzerland. (2012).

24. Feil EJ, Li BC, Aanensen DM, Hanage WP, Spratt BG. eBURST: inferring patterns of evolutionary descent among clusters of related bacterial genotypes from multilocus sequence typing data. J Bacteriol. (2004) 186:1518-30. doi: 10.1128/JB.186.5.1518-1530.2004

25. Amemura-Maekawa J, Kikukawa K, Helbig JH, Kaneko S, Suzuki-Hashimoto A, Furuhata K, et al. Distribution of monoclonal antibody subgroups and sequence-based types among Legionella pneumophila serogroup 1 isolates derived from cooling tower water, bathwater, and soil in Japan. Appl Environ Microbiol. (2012) 78:4263-70. doi: 10.1128/AEM.06869-11

26. Lee HK, Shim JI, Kim HE, Yu JY, Kang YH. Distribution of Legionella species from environmental water sources of public facilities and genetic diversity of L. pneumophila serogroup 1 in South Korea. Appl Environ Microbiol. (2010) 76:6547-54. doi: 10.1128/AEM.00422-10

27. Hunter PR, Gaston MA. Numerical index of the discriminatory ability of typing systems: an application of Simpson's index of diversity. J Clin Microbiol. (1988) 26:2465-6. doi: 10.1128/JCM.26.11.2465-2466.1988

28. Valavane A, Chaudhry R, Malhotra P. Multiplex polymerase chain reaction of genetic markers for detection of potentially pathogenic environmental Legionella pneumophila isolates. Indian J Med Res. (2017) 146:392. doi: 10.4103/ijmr.IJMR_623_16

29. Graells T, Hernández-García M, Pérez-Jové J, Guy L, Padilla E. Legionella pneumophila recurrently isolated in a Spanish hospital: two years of antimicrobial resistance surveillance. Environ Res. (2018) 166:638-46. doi: 10.1016/j.envres.2018.06.045

30. Laganà $\mathrm{P}$, Facciolà A, Palermo R, Delia. S. Environmental surveillance of legionellosis within an Italian university hospital-results of 15 years of analysis. Int J Environ Res Public. Health. (2019) 16:1103. doi: 10.3390/ijerph16071103

31. Liu WK, Healing DE, Yeomans JT, Elliott TS. Monitoring of hospital water supplies for Legionella. J Hosp Infect. (1993) 24:1-9. doi: 10.1016/0195-6701(93)90084-D

32. Sabria M, Modol JM, Garcia-Nunez M, Reynaga E, Pedro-Botet ML, Sopena N, et al. Environmental cultures and hospital-acquired Legionnaires' disease: a 5-year prospective study in 20 hospitals in Catalonia, Spain. Infect Control Hosp Epidemiol. (2004) 25:1072-6. doi: 10.1017/S01959417000 $7750 \mathrm{X}$

33. Yu PY, Lin YE, Lin WR, Shih HY, Chuang YC, Ben RJ, et al. The high prevalence of Legionella pneumophila contamination in hospital potable water systems in Taiwan: implications for hospital infection control in Asia. Int. J Infect Dis. (2008) 12:416-20. doi: 10.1016/j.ijid.2007. 11.008

34. Lin YE, Stout JE, Victor LY. Controlling Legionella in hospital drinking water: an evidence-based review of disinfection methods. Infect Control Hosp Epidemiol. (2011) 32:166-73. doi: 10.1086/657934

35. Katsiaflaka A, Pournaras S, Kristo I, Mouchtouri VA, Kyritsi M, Velonakis E, et al. Epidemiological investigation of Legionella pneumophila serogroup 2 to 14 isolates from water samples by amplified fragment length polymorphism and sequence-based typing and detection of virulence traits. Appl Environ Microbiol. (2016) 82:6102-8. doi: 10.1128/AEM. 01672-16 
36. Zeng LZ, Liao HY, Luo LZ, He SS, Tian QI, Zhou HJ, et al. An investigation on the molecular characteristics and intracellular growth ability among environmental and clinical isolates of Legionella pneumophila in Sichuan Province, China. Biomed Environ Sci. (2019) 32:520-30. doi: 10.3967/bes2019.069

37. Goh KT, Ng DL, Yap J, Ma S, Ooi EE. Surveillance, prevention, and control of legionellosis in a tropical city-state. Am J Infect Control. (2005) 33:286-91. doi: 10.1016/j.ajic.2004.12.001

38. Reimer AR, Au S, Schindle S, Bernard KA. Legionella pneumophila monoclonal antibody subgroups and DNA sequence types isolated in Canada between 1981 and 2009: laboratory component of national surveillance. Eur J Clin Microbiol Infect Dis. (2010) 29:191-205. doi: 10.1007/s10096-009-0840-3

39. Kozak NA, Benson RF, Brown E, Alexander NT, Taylor TH, Shelton BG, et al. Distribution of lag-1 alleles and sequence-based types among Legionella pneumophila serogroup 1 clinical and environmental isolates in the United States. J Clin Microbiol. (2009) 47:2525-35. doi: 10.1128/JCM.02410-08

40. Amemura-Maekawa J, Kura F, Chida K, Ohya H, Kanatani JI, Isobe J, et al. Legionella pneumophila and other Legionella species isolated from legionellosis patients in Japan between 2008 and 2016. Appl Environ Microbiol. (2018) 84:e00721-18. doi: 10.1128/AEM.00721-18
41. Harrison TG, Fry NK, Afshar B, Bellamy W, Doshi N, Underwood AP. Typing of Legionella pneumophila and its role in elucidating the epidemiology of legionnaire's disease. In Cianciotto NP, Kwaik YA, Edelstein PH, Fields BS, Geary DF, Harrison TG, et al. editors. Legionella: State of the Art 30 Years After Its Recognition. Washington, DC: ASM Press. (2005). p. 94-99. doi: 10.1128/9781555815660.ch25

42. Quero S, Párraga-Niño N, Sabria M, Barrabeig I, Sala MR, Jané M, et al. Legionella SBT applied directly to respiratory samples as a rapid molecular epidemiological tool. Sci Rep. (2019) 9:1-6. doi: 10.1038/s41598-018-36924-w

Conflict of Interest: The authors declare that the research was conducted in the absence of any commercial or financial relationships that could be construed as a potential conflict of interest.

Copyright (c) 2020 Sreenath, Chaudhry, Vinayaraj, Dey, Kabra, Thakur and Guleria. This is an open-access article distributed under the terms of the Creative Commons Attribution License (CC BY). The use, distribution or reproduction in other forums is permitted, provided the original author(s) and the copyright owner(s) are credited and that the original publication in this journal is cited, in accordance with accepted academic practice. No use, distribution or reproduction is permitted which does not comply with these terms. 